\title{
Economic methods for valuing the outcomes of genetic testing: beyond cost-effectiveness analysis
}

Scott D. Grosse, $P h D^{1}$, Sarah Wordsworth, $P h D^{2}$, and Katherine Payne, $P h D^{3}$

\begin{abstract}
Genetic testing in health care can provide information to help with disease prediction, diagnosis, prognosis, and treatment. Assessing the clinical utility of genetic testing requires a process to value and weight different outcomes. This article discusses the relative merits of different economic measures and methods to inform recommendations relative to genetic testing for risk of disease, including cost-effectiveness analysis and costbenefit analysis. Cost-effectiveness analyses refer to analyses that calculate the incremental cost per unit of health outcomes, such as deaths prevented or life-years saved because of some intervention. Cost-effectiveness analyses that use preference-based measures of health state utility such as quality-adjusted life-years to define outcomes are referred to as cost-utility analyses. Cost-effectiveness analyses presume that health policy decision makers seek to maximize health subject to resource constraints. Cost-benefit analyses can incorporate monetary estimates of willingness-to-pay for genetic testing, including the perceived value of information independent of health outcomes. These estimates can be derived from contingent valuation or discrete choice experiments. Because important outcomes of genetic testing do not fit easily within traditional measures of health, costeffectiveness analyses do not necessarily capture the full range of outcomes of genetic testing that are important to decision makers and consumers. We recommend that health policy decision makers consider the value to consumers of information and other nonhealth attributes of genetic testing strategies. Genet Med 2008:10(9):
\end{abstract} 648-654.

Key Words: cost-effectiveness, economic evaluation, genetics, value of information

The use of genetic information in health care has both clinical and economic implications. Genetic information may be useful to various stakeholders, such as health care system decision makers (hereafter referred to as "decision makers"), practicing clinicians, and patients and their families. Genetic testing is a complex intervention that can be used to predict the risk of developing a condition; facilitate more rapid and accurate diagnoses of genetic conditions; and, by leading to earlier or more precise interventions, potentially prevent disease, prolong life, and promote health. Genetic information can aid individual and family decision-making even if the results of a genetic test do not affect clinical management or lead to a measurable effect on health. ${ }^{1}$ However, genetic testing also can lead

From the ${ }^{1}$ National Center on Birth Defects and Developmental Disabilities, Coordinating Center for Health Promotion, Centers for Disease Control and Prevention, Atlanta, Georgia; ${ }^{2}$ Health Economics Research Centre, The University of Oxford, Oxford, United Kingdom; and ${ }^{3}$ Health Economics, Health Methodology Research Group, The University of Manchester and Nowgen - A Centre for Genetics in Healthcare, Manchester, United Kingdom.

Scott D. Grosse, PhD, Centers for Disease Control and Prevention, 1600 Clifton Road NE, Mail Stop E-87, Atlanta, GA 30333.E-mail: SGG4@CDC.GOV.

The findings and conclusions in this report are those of the authors and do not necessarily represent the official position of the Centers for Disease Control and Prevention.

Disclosure: The authors declare no conflict of interest.

Submitted for publication December 18, 2007.

Accepted for publication June 11, 2008.

DOI: $10.1097 /$ GIM.0b013e3181837217 to follow-on testing that is invasive and expensive or the initiation of management strategies of uncertain benefit, and result in negative psychosocial effects such as fear of discrimination and complacency from negative test results that might encourage unhealthy behaviors..$^{2-4}$

Genetic-based technologies offer the prospect of information to guide clinical decision-making but will also have impacts on the use of health care resources. Assessing the clinical utility of genetic testing requires a process to value and weight different outcomes. ${ }^{5,6}$ In this article, we define outcomes as the effects of a health care service or intervention, which include both health outcomes such as disease or quality of life and nonhealth outcomes such as waiting time for test results or type of care delivered. Robust evidence on the economic impact of genetics tests might be considered a prerequisite to informed decision-making. Economic evaluation methods that value multiple outcomes are important to inform health policy decisions. Economic evaluations typically focus on outcome measures such as the numbers of cases of disease detected or deaths prevented; such evaluations are termed costeffectiveness analyses (CEAs). Albert Einstein famously said, "not all that counts can be counted."

The primary objective of this article was to describe how by going "beyond" cost-effectiveness analysis, it is possible to "count" additional outcomes. Specifically, this article aims to describe and discuss alternative economic methods for assess- 
ing the outcomes of predictive and diagnostic molecular genetic testing using tests of demonstrated analytic and clinical validity. We do not include pharmacogenetic testing, which is used to predict treatment response and inform the choice of medicine or dosing regimen, because it raises different issues in terms of measuring outcomes and impacts on the individual and family members. Likewise, we do not address applications of genetic testing outside the health care setting, such as consumer-oriented genetic profiling based on putative gene-disease associations, or the potential benefits and risks from technologies such as whole-genome sequencing. We briefly review different types of economic evaluation methods and their applications to genetic testing in health care.

\section{ECONOMIC METHODS FOR THE EVALUATION OF OUTCOMES}

Economic evaluation methods that compare costs and outcomes can be classified into two main types, CEA and costbenefit analysis (CBA). The most obvious difference is that in CBA outcomes are all expressed in monetary units and in CEA the difference in costs between two interventions is compared with the difference in health outcomes. A more fundamental difference is that a CEA provides information as to whether an intervention maximizes the health of a population in a resource-constrained health system whereas a CBA aims to determine whether social welfare, including both health and nonhealth outcomes and values, is maximized subject to societal budget constraints. CBA is used widely outside of health care, particularly in the regulatory arena, ${ }^{7}$ whereas CEA is more common in health care. ${ }^{8-11}$ CEAs often take the perspective of the health care system or payer, with only costs incurred by health care providers and payers included, although it is often recommended that CEAs take the societal perspective, including costs and benefits to consumers of health care. ${ }^{12} \mathrm{~A}$ decision-maker could potentially use CBA results to compare between health and nonhealth programs and to assess outcomes of health programs other than mortality, morbidity, and health-related quality of life. Using the results of a CBA might be problematic if a decision-maker wishes to focus only on maximizing health gain.

CEAs are used to compare the efficiency of strategies that differ in effectiveness and cost. Interventions that are both more effective in improving total health outcomes and cost less than an alternative are said to be dominant and the optimal intervention should be clear to a decision maker developing local or national policies. In situations in which a proposed new intervention has increased costs and improved outcomes relative to another strategy it becomes necessary to calculate the incremental cost-effectiveness ratio (ICER) between that pair of strategies. The ICER is the ratio in which the incremental costs are divided by the incremental outcomes. Decision makers must decide whether a particular ICER is indicative of acceptable value for their local or national context and available budget.
An important question is whether health care decision makers care only about health outcomes, as is assumed in CEA, or take a broader interest in societal welfare. ${ }^{13-15}$ It has been argued that ethical and distributional questions need to be considered by decision makers in addition to economic efficiency. ${ }^{16,17}$ This presumes that health care decision makers actually pay attention to ICERs, which is likely to differ across health care settings and political jurisdictions. US health care decision makers are often reported to not make cost-effectiveness an explicit consideration, ${ }^{11}$ whereas the National Institute for Health and Clinical Excellence in the United Kingdom requires it. ${ }^{18}$

CEAs commonly calculate the cost per unit of "natural" health outcomes, e.g., deaths prevented or life-years saved per unit of cost. Another type of CEA, cost-utility analysis (CUA), uses preference-based measures of health state utility such as quality-adjusted life-years (QALYs). ${ }^{15}$ The US Panel on CostEffectiveness in Health and Medicine and National Institute for Health and Clinical Excellence both recommend that CEAs use QALYs as the outcome measure, ${ }^{12,19}$ using CEA to encompass both CUA and CEA. Economic evaluations of diagnostic testing applications often focus on estimating the cost per additional case identified, because of the difficulties in translating outcomes to life-years or QALYs. ${ }^{20}$

QALYs are calculated based on the perceived well-being or "utilities" respondents assign to different health states, and reflect preferences or trade-offs between health states and death. ${ }^{21}$ An interval scale is used to describe a utility weight, where a score of " 1 " is said to represent "perfect" health and " 0 " represents death. The number of QALYs is calculated as the sum of the duration (number of years) spent in each health state multiplied by the proportional utility weight between 0 and 1 for that health state. Preference weights for the calculation of QALYs can be estimated using direct or indirect methods. Direct methods typically ask individuals, whether current patients or members of the public, to either trade off the probability of being returned to a state of full health with the probability of death (standard gamble) or of being returned to a state of full health for a given duration of time with the number of years of life that would be given up at some point in the future (time trade-off).

Commonly, generic multiattribute instruments are used to assess QALY weights. These multiattribute instruments ask individuals to give ratings to different dimensions of their life (for example, level of pain, mobility, and ability to perform day-to-day activities) for a defined period (for example, today or last month). The rating scores are combined with preference scores derived from population-based samples using time trade-off and other methods to calculate utility weights. An example is the EQ-5D (or EuroQol), which comprises five domains: mobility, self-care, usual activities, pain or discomfort, and anxiety or depression. ${ }^{22}$ Typically, individuals with a given condition are asked to complete the EQ-5D before and after they have received an intervention under evaluation. The changes in utility weights are then compared with changes over time among a sample of patients who have not received the 
intervention. The net changes in utility weights are used to calculate the QALYs gained for the new intervention. Countryspecific recommendations in the United States and United Kingdom suggest the use of population-based utility weights. ${ }^{12,19}$ However, utilities estimated by members of the public with no experience of the health states do not necessarily accurately reflect the preferences of people who have experience of those health states. ${ }^{23}$

CUAs have limitations for the economic evaluation of genetic tests and use of genetic information. First, QALYs are typically calculated only for the directly affected individual, excluding family members, ${ }^{12,21}$ with the exception of prenatal testing. ${ }^{24,25}$ Effects on other family members, which have been referred to as family spillover benefits, ${ }^{26}$ might be particularly important in genetic testing because knowledge of a genetic risk carries potential harm and benefit to family members. ${ }^{27}$ In particular, QALYs as measured using existing generic multiattribute instruments do not include nonhealth outcomes, which could include information to aid decision-making and empowerment ${ }^{28}$ or "process" attributes of care, such as the nature of the consultation (e.g., offering time to talk), the location of care, the waiting time for results and the nature of any follow-up care, or whether care is coordinated and tailored to the family. ${ }^{29-31}$ Theoretically, it is possible to incorporate process attributes into a QALY measure using standard gamble or time trade-off methods, but in practice this is likely to require the development of new or adapted methods. ${ }^{32}$

Nonhealth outcomes are relevant to economic evaluation from the societal perspective but may not be as relevant to health care decision makers. Health care providers, particularly in genetic services, are reported to value informed decision-making, ${ }^{33}$ but health care payers, at least in the United States, are reported to not take into account the value of diagnostic information to health care consumers. ${ }^{34}$

In CBA, outcomes are expressed in monetary terms and subtracted from costs to estimate net benefits. The decision rule to select the optimal intervention is that if the net benefit is positive (benefits outweigh costs), the intervention is justified in terms of increasing societal welfare. One approach to attaching monetary values to health outcomes is to estimate the direct costs of care or the "indirect" or "human capital" costs of lost economic production associated with morbidity and premature mortality that are calculated to be avoided by the intervention. ${ }^{35} \mathrm{~A}$ second approach, which is favored by most economists, is the use of estimates of consumer "willingness-to-pay" (WTP) (or "willingness-to-accept") to value benefits in CBA. ${ }^{7,36-38}$ Methods to obtain WTP estimates are discussed below.

A CBA of a genetic test could be used to go beyond CEA, that by definition focuses on maximizing health gain, and use estimates of WTP to derive monetary valuations of outcomes can readily encompass both health and nonhealth outcomes and attributes. ${ }^{36-39}$ For example, much of the value to consumers of routine ultrasound in pregnancy, as revealed by WTP, is the value of information unrelated to its use in medical management. ${ }^{40}$ Many parents place a positive value on knowing the diagnosis in a young child of an X-linked condition, such as Duchenne muscular dystrophy. ${ }^{41}$ A CBA could use WTP values elicited from parents regarding testing for a condition for which treatment does not necessarily prevent mortality or morbidity. Such values would reflect the possible utility or disutility of the information in of itself.

WTP estimates can be derived using different methods, including elicitation of revealed preferences (using actual market data observing what people "buy") or stated preferences (what people say they prefer) through surveys. Revealed preference data can only be used for health care interventions that are already in practice. Stated preference methods allow estimation of preferences for new services yet to be introduced.

In one stated preference method, the maximum amount of money an individual is willing to pay is obtained via a contingent valuation $(\mathrm{CV})$ questionnaire in which a respondent is asked whether he or she would be willing to pay a specified amount of money for an intervention to prevent, diagnose, or manage a condition (the health care scenario). There are a number of issues in the application of CV studies, particularly among health care systems in which patients do not normally pay for care at the point of use. WTP studies require careful design in questionnaire development and method of survey administration. How the respondent is asked to state his or her WTP can influence whether the method results in valid and reliable monetary valuation estimates for decision-making. ${ }^{42}$ Other issues include the dependence of WTP estimates on household income and on how questions are posed. ${ }^{9,42-44}$ The potential of WTP estimates to inform health care policy remains largely unfulfilled mainly because of difficulties with applying the technique in practice; "most of the published CV studies [in health and health policy applications] are experimental in nature, attempting to explore measurement feasibility issues rather than being full program evaluations using CBA." 15

An alternative to $\mathrm{CV}$ is conjoint analysis, in which respondents are asked to rank, rate, or choose an intervention from multiple scenarios that describe health care intervention or service characteristics in order of preference. It goes beyond CV by obtaining information on the relative value of specific attributes of health care rather than just for the health care intervention or service as a package. Conjoint analysis is a stated preference technique that originated in mathematical psychology and has been widely used in market research, ${ }^{45}$ and by economists to value preferences in areas such as transport, environmental, and health care economics. There are different types of conjoint analysis. Transport economists developed discrete choice experiments (DCEs) that are a form of conjoint analysis in which respondents make a "discrete choice" between two or more interventions or services that differ with respect to certain predefined attributes. ${ }^{46}$ When DCEs were first used in health care the term "conjoint analysis" was inappropriately used interchangeably. The term DCE refers to a design that includes a discrete choice rather than rating or ranking type experiment. We prefer to use the term DCE because this approach is currently more widely used in health 
care. The design issues associated with $\mathrm{CV}$ are also relevant to DCEs. ${ }^{47}$

DCEs, with the inclusion of a cost attribute that allows the indirect estimation of WTP, have been used by health economists. ${ }^{31,48-50}$ In addition to the WTP to avert a specified outcome, such as major depression, this method has also been used to quantify the WTP to avoid side effects of treatment, e.g., from antidepressants. ${ }^{51}$ However, the increased use of DCEs in health care has not yet been matched by a corresponding increase in health care CBAs using DCE-derived valuations. ${ }^{52}$ The value of DCE studies to date lies more in identifying the attributes of services that most affect people's preferences and using this information to tailor interventions than in directly informing decision-making on whether an intervention is justified on economic grounds.

A few studies have used DCEs to examine the informational value of diagnostic testing. One such study examined the perceived value of magnetic resonance imaging in knee injury, which can influence therapeutic choice but has not been shown to have long-term health benefits. ${ }^{53}$ In a conjoint analysis of cardiac risk assessment strategies, people in both Great Britain and Italy placed a high value on prognostic information, with roughly equal values on reducing uncertainty in the prediction of risk and actual reduction in risk. ${ }^{54}$ Physicians in both countries placed a lower value on prognostic information, on average one third as high.

\section{GENETIC TESTING APPLICATIONS}

Four systematic reviews of economic evaluations of genetic testing in general have been published in recent years. ${ }^{39,55-57}$ Two reviews were restricted to predictive molecular genetic tests ${ }^{55,57}$ and the other two reviews covered biochemical, cytogenetic, and molecular testing. ${ }^{39,56}$ The reviews covered different time periods and used different search criteria and databases. Despite differences, each showed that CEAs and partial economic evaluations that report data on costs and intermediate outcomes (e.g., cost per case detected) predominate in clinical genetics. The exception was for prenatal testing, for which CBAs restricted to human capital or averted medical costs as the measure of benefit have remained common. ${ }^{58,59}$ Relatively few CUAs and no WTP-based CBAs of genetic testing applications were identified.

Mutation testing for familial cancers has been assessed in a number of economic evaluations. Sensitivity analyses in these studies suggest that the cost-effectiveness of testing depends on the frequency of mutations in the target population, the uptake of efficacious treatments, and the alternative with which testing is compared. For example, Tengs and Berry ${ }^{60}$ calculated that $B R C A$ testing of the general US population would be regarded as not cost-effective at $\$ 1.6$ million per QALY. They calculated that testing of women at moderately elevated risk, with a $5 \%$ probability of a mutation, would cost $\$ 34,000$ per QALY, which generally would be regarded as cost-effective. The QALY estimates in that study were accounted for chiefly by gains in survival from prophylactic surgery, although they also took into account potential harms of prophylactic surgery through reductions in QALY weights.

A limitation of CEA/CUA studies of testing for inherited cancer mutations is the assumption that the value of mutation testing derives solely from the use of test results to reduce the risk of death through prophylactic surgery or chemoprevention. ${ }^{61}$ It is known from other contexts that diagnostic or predictive testing can have both positive and negative informational utility independent of therapeutic choice, depending on individual preferences and test results. ${ }^{62,63}$ Women who choose to be tested for BRCA mutations often seem to be motivated more by the opportunity to benefit family members than themselves by making it easier for relatives to learn their own genetic risk. ${ }^{64}$ Women who had no intention of using either prophylactic surgery or chemoprevention to reduce their risk of developing cancer in one study appeared just as likely to undergo mutation testing. ${ }^{65}$ Presumably, many of those women chose testing for $B R C A$ mutations because they thought that it could help them to reduce the risk of dying from cancer through increased surveillance (early detection), to inform relatives, to increase self-knowledge, or all of the above. However, conclusive evidence that increased surveillance in mutation carriers reduces risk of mortality is lacking. ${ }^{66}$

The psychosocial consequences of predictive genetic testing have been best studied in Huntingdon disease (HD). One large study that tracked more than 200 individuals for 5 years after mutation testing found that most people in HD families tested experienced reduced psychological distress, regardless of whether they tested positive or negative, although 14 of 202 experienced clinically significant adverse events within 2 years of testing. ${ }^{67}$ Intergenerational impacts of disclosure of $\mathrm{HD}$ genetic risk have also been studied. ${ }^{68}$ However, the information value of predictive testing for HD has not been incorporated into economic models.

Others have noted the exclusion of psychosocial and intergenerational issues from most economic evaluations, whether specific to genetics ${ }^{33}$ or generic. ${ }^{22}$ Similarly, others have argued that economic evaluations of prenatal testing should consider a broader scope of utility. ${ }^{26,57} \mathrm{Few}$ economic evaluations in health care in general, let alone in genetics, have attempted to measure or value family-level psychosocial outcomes, such as reassurance from an accurate test result and "spillover" effects on family members. ${ }^{26}$ In contrast, the majority of the genetics literature evaluating the effect of genetics services and associated testing has tended to use psychosocial measures of anxiety, depression, and effects on mood. ${ }^{1}$

In addition to their use in CUAs to inform decision makers about whether an intervention provides good value for money, QALYs based on patient preferences can potentially be used to inform clinical decisions. For women at high risk of having a $B R C A$ mutation, prophylactic mastectomy and oophorectomy are estimated to reduce by $85-100 \%$ the risks of breast cancer and ovarian cancer, respectively. ${ }^{66}$ However, both types of surgery can have negative effects on quality of life. Different strategies can maximize survival or utility, depending on treatment preferences. For example, one study calculated that for the typ- 
ical BRCA mutation carrier combined mastectomy and oophorectomy would maximize life expectancy whereas oophorectomy alone would maximize QALYs. ${ }^{69}$ Similarly, for most but not all individuals with Lynch syndrome (mutation carriers for hereditary nonpolyposis colorectal cancer), prophylactic colectomy would maximize survival whereas surveillance by colonoscopy would maximize QALYs. ${ }^{70}$ Because the optimal treatment strategy for individuals can differ based on their preferences, individual preferences should be elicited and used in shared decision-making. ${ }^{71,72}$

An important question for applications of genetic testing in diagnosis or prognosis is whether the instruments used to calculate QALY weights pick up all the important attributes associated with the test. In particular, existing survey instruments for calculating QALY weights might contain insufficient domains to measure all the potential outcomes from a genetic testing service, such as the ability to make an informed life decision. ${ }^{33}$ One study of nongenetic diagnostic testing concluded that the potential psychological benefits of reassurance from a negative diagnostic test in patients with equivocal signs lowered the incremental cost-effectiveness ratio of testing from more than $\$ 100,000$ per QALY to approximately $\$ 15,000$ per QALY. ${ }^{73}$ A recent study of palliative treatments for esophageal cancer adjusted QALYs for process utility but found little impact on the relative ranking of different strategies. ${ }^{32}$

CBAs can incorporate psychosocial factors that consider other benefits or harms. WTP is able to capture negative psychosocial effects associated with genetic testing. An individual's WTP for testing is an indication of how much they value a particular good or service. A genetic test can either raise or lower anxiety depending on the results of the test. The expected value of the test to the individual consumer reflects both the expected probability of each type of result and the positive and negative emotions that would be expected to ensue. Previous CBAs of prenatal screening have made the assumption that the only quantifiable benefit is the opportunity to terminate an affected pregnancy. ${ }^{30}$ Payne et al..$^{33}$ explored patients' and health care professionals' views about the value of other types of patient benefits from genetic services and testing.

A recent CV study assessed WTP for a hypothetical genetic test for assessing risk of hereditary colorectal cancer. The mean and median WTP for a general US online sample was $\$ 150$, although $17 \%$ were unwilling to pay any amount. ${ }^{74}$ Although the self-reported intention to obtain screening for colorectal cancer was strongly positively associated with WTP, as was family income, fewer than half the sample indicated that they would be more likely to seek colorectal cancer screening if they were found to be at elevated risk. Anyone who expressed a positive WTP and would not plan to take preventive action based on the results of the test implicitly places a positive net value on the test result information per se.

CV studies have also been used to explore preferences for alternative methods of prenatal screening for cystic fibrosis carrier status. ${ }^{75} \mathrm{CV}$ studies of prenatal screening have reported that WTP estimates are not very sensitive to whether a woman plans to terminate an affected pregnancy or not, indicating that some of the perceived value of prenatal diagnosis comes from benefits other than avoiding the birth of an affected fetus. ${ }^{76-78}$ Further research is necessary to identify and quantify the nature of these other benefits of genetic testing from the perspective of the individuals to whom testing is offered. ${ }^{72}$

In genetics, DCEs have been applied in prenatal testing ${ }^{79}$ and in genetic counseling in a cancer genetics service. ${ }^{80}$ Hall et al. ${ }^{81}$ used a DCE to investigate consumer preferences for genetic carrier testing in Tay-Sachs disease and cystic fibrosis. The DCE comprised 12 attributes and focused on the uptake of carrier testing based on provision of information before conception and presented individuals with a decision to choose more than one genetic test, which is a reflection of clinical practice. Respondents were more likely to accept testing if there was a higher risk of disease, when the accuracy of the test improved, when their doctor recommended testing, and when the price of the test decreased.

A further advantage of DCEs, in the context of genetic testing, is that they are able to evaluate both current and future tests. Because of the ability to include hypothetical scenarios, factors influencing the potential uptake (demand) for a new genetic test could be considered before the test is placed into routine clinical practice. ${ }^{81}$ An obvious limitation of hypothetical scenarios is that stated preferences do not necessarily equate with actions if faced with real-world choices. However, a recent conjoint analysis of a Canadian choice-format survey of preferences for screening for colorectal cancer, including new and hypothetical methods, generated findings consistent with observed behaviors: one third of respondents would not choose any screening modality and the most widely available method was the least favored; cost had little effect on choices among screening methods. ${ }^{82}$

\section{CONCLUSION}

The large majority of economic evaluations of health care interventions, including genetic tests outside of prenatal testing, have been CEAs taking the health care system perspective. Although these can be useful for prioritizing health care resources, they are inadequate for maximizing welfare over both health and nonhealth outcomes. In this article, we have argued that CBA methods, especially DCEs, are necessary to estimate the full value of genetic testing. Because many important outcomes or attributes of genetic testing do not fit easily within traditional measures of health, CEAs do not necessarily capture the full range of outcomes that are important to health care decision makers and consumers and, hence, can provide incomplete information. Although all economic evaluation methods, including CBA, are subject to methodological limitations, we believe that the use of CV, DCE, and CBA is an important step forward in fully evaluating this area of health care, especially given the limitations of CEA in addressing several aspects of genetic testing. 


\section{ACKNOWLEDGMENTS}

The authors acknowledge helpful comments from Laura Beskow, Ralph Coates, James Gudgeon, Jane Karwoski, Kathryn Phillips, and Janet Williams.

An earlier version of this article was presented at the ESRC Seminar on the Economics of Genetic Testing, University of Oxford, November 2006.

\section{References}

1. Payne K, Nicholls S, McAllister M, MacLeod R Donnai D, Davies LM. Outcome measurement in clinical genetics services: a systematic review of validated measures Value Health 2008;11:497-508.

2. Holtzman NA, Watson MS, editors. Promoting safe and effective genetic testing in the United States. Final report of the Task force on Genetic Testing. 1997. Available at: http://www.genome.gov/10001733. Accessed June 22, 2005.

3. Col NF. The use of gene tests to detect hereditary predisposition to chronic disease: is cost-effectiveness analysis relevant? Med Decis Making 2003;23:441-448.

4. Sanderson S, Zimmern R, Kroese M, Higgins J, Patch C, Emery J. How can the evaluation of genetic tests be enhanced? Lessons learned from the ACCE framework and evaluating genetic tests in the United Kingdom. Genet Med 2005;7:495-500.

5. Grosse SD, Khoury MJ. What is the clinical utility of genetic testing? Genet Med 2006;8:448-450.

6. Burke W, Atkins D, Gwinn M, et al. Genetic test evaluation: information needs of clinicians, policy makers, and the public. Am J Epidemiol 2002;156:311-318.

7. Krupnick AJ. Valuing health outcomes: policy choices and technical issues. Washington, DC: Resources for the Future, 2004. Available at http://www.rff.org/rff/ Documents/RFF-RPTValuingHealthOutcomes.pdf. Accessed April 25, 2006.

8. Oliver A, Healy A, Donaldson C. Choosing the method to match the perspective: economic assessment and its implications for health services efficiency. Lancet 2002; 359:1771-1774.

9. Cookson R. Willingness to pay methods in health care: a skeptical view. Health Econ 2003;12:891-894.

10. Neumann PJ. Using cost-effectiveness analysis to improve health care. New York: Oxford University Press, 2005.

11. Grosse SD, Teutsch SM, Haddix AC. Lessons from cost-effectiveness research for United States public health policy. Annu Rev Pub Health 2007;28:365-391.

12. Gold MR, Siegel JE, Russell LB, Weinstein MC, editors. Cost-effectiveness in health and medicine. New York: Oxford University Press, 1996.

13. Hurley J. Welfarism, extra-welfarism and evaluative economic analysis in the health sector. In: Barer M, Getzen T, Stoddart G, editors. Health, health care and health economics: perspectives on distribution. Chichester: Wiley, 1998:373-395.

14. Birch S, Donaldson C. Valuing the benefits and costs of health care programmes: where's the 'extra' in extra-welfarism? Soc Sci Med 2003;56:1121-1133.

15. Drummond MF, Sculpher MJ, Torrance GW, O’Brien BJ, Stoddart GL. Methods for the economic evaluation of health care programmes, 3rd ed. Oxford: Oxford University Press, 2005.

16. Coast J. Is economic evaluation in touch with society's health values? BMJ 2004;329: 1233-1236.

17. Sassi F, Le Grand J, Archard L. Equity versus efficiency: a dilemma for the NHS. If the NHS is serious about equity it must offer guidance when principles conflict. BMJ 2001;323:762-763.

18. Buxton MJ. Economic evaluation and decision making in the UK. Pharmacoeconomics 2006;24:1133-1142.

19. National Institute for Health and Clinical Excellence. Guide to the methods of technology appraisal. Reference N0515. London. 2004. Available at: http://www. nice.org.uk/aboutnice/howwework/devnicetech/technologyappraisalprocessguides/ guide_to_the_methods_of_technology_appraisal_reference_n0515.jsp. Accessed October $8,2007$.

20. Mushlin AI, Ruchlin HS, Callahan MA. Cost-effectiveness of diagnostic tests. Lancet 2001;358:1353-1355.

21. Neumann PJ, Goldie SJ, Weinstein MC. Preference-based measures in economic evaluation in health care. Annu Rev Public Health 2000;21:587-611.

22. Dolan P. Modeling valuations for EuroQol health states. Med Care 1997;35:1095-1108.

23. Brazier J, Akehurst R, Brennan A, et al. Should patients have a greater role in valuing health states? Appl Health Econ Health Policy 2005;4:201-208.

24. Harris RA, Washington AE, Nease RF Jr, Kuppermann M. Cost utility of prenatal diagnosis and the risk-based threshold. Lancet 2004;363:276-282.

25. Musci TJ, Caughey AB. Cost-effectiveness analysis of prenatal population-based fragile X carrier screening. Am J Obstet Gynecol 2005;192:1905-1912.

26. Basu A, Meltzer D. Implications of spillover effects within the family for medical cost-effectiveness analysis. J Health Econ 2005;24:751-773.
27. McAllister M, Payne K, Nicholls S, Macleod R, Donnai D, Davies LM. Improving service evaluation in clinical genetics: identifying effects of genetic diseases on individuals and families. J Genet Counsel 2007;16:71-83.

28. McAllister M, Payne K, Macleod R, et al. Patient empowerment in clinical genetics services. J Health Psychol. In press.

29. Mooney G. Key issues in health economics. London: Harvester Wheatsheaf, 1994.

30. Mooney G, Lange M. Ante-natal screening: what constitutes 'benefit'? Soc Sci Med 1993;37:873-878.

31. Ryan M. Using conjoint analysis to go beyond health outcomes: an application to in vitro fertilisation. Soc Sci Med 1999;48:535-546.

32. McNamee P, Seymour J. Incorporation of process preferences within the QALY framework: a study of alternative methods. Med Decis Making 2008;28:443-452.

33. Payne K, Nicholls S, McAllister M, et al. Outcome measures for clinical genetics services: a comparison of genetics healthcare professionals and patients' views. Health Policy 2007;84:112-122.

34. Hirth RA, Bloom BS, Chernew ME, Fendrick AM. Patient, physician, and payer perceptions and misperceptions of willingness to pay for diagnostic certainty. Int $J$ Technol Assess Health Care 2000;16:35-49.

35. Waitzman NJ, Scheffler RM, Romano PS. The cost of birth defects. Lanham, MD: University Press of America, 1996.

36. Donaldson C, Currie G, Mitton C. Cost effectiveness analysis in health care: contraindications. BMJ 2002;325:891-894.

37. Gafni A. Using willingness-to-pay as a measure of benefits: what is the relevant question to ask in the context of public decision making? Med Care 1991;29:12461252.

38. O'Brien B, Viramontes JL. Willingness to pay: a valid and reliable measure of health state preference? Med Decis Making 1994;14:289-297.

39. Jarrett J, Mugford M. Genetic health technology and economic evaluation: a critical review. Appl Health Econ Health Policy 2006;5:27-35.

40. Berwick DM, Weinstein MC. What do patients value? Willingness to pay for ultrasound in normal pregnancy. Med Care 1985;23:881-893.

41. Bailey DB Jr, Beskow LM, Davis AM, Skinner D. Changing perspectives on the benefits of newborn screening. Ment Retard Dev Disabil Res Rev 2006;12:270-279.

42. Smith RD. Construction of the contingent valuation market in health care: a critical assessment. Health Econ 2003;12:609-628.

43. Watson V, Ryan M. Exploring preference anomalies in double bounded contingent valuation. J Health Econ 2007;26:463-482.

44. Smith RD. Contingent valuation in health care: does it matter how the 'good' is described? Health Econ 2008;17:607-617.

45. Green PE, Srinivasan V. Conjoint analysis in marketing: new developments with implications for research and practice. J Market 1990;54:3-19.

46. Hensher DA. Stated preference analysis of travel choices: the state of practice. Transportation 1994;21:107-133.

47. Ratcliff J. The use of conjoint analysis to elicit willingness-to-pay values. Proceed with caution? Int J Technol Assess Health Care 2000;16:270-280.

48. van der Pol M, Cairns J. Establishing patient preferences for blood transfusion support: an application of conjoint analysis. J Health Serv Res Policy 1998;3:70-76.

49. Ryan M, Gerard K. Using discrete choice experiments in health economics: moving forward. In: Scott A, Maynard A, Elliott RF, editors. Advances in health economics. Chichester: Wiley, 2003.

50. Wordsworth S, Ryan M, Skatun D, Waugh N. Women's preferences for cervical cancer screening: a study using a discrete choice experiment. Int J Technol Assess Health Care 2006;22:344-350.

51. Morey E, Thacher JA, Craighead WE. Patient preferences for depression treatment programs and willingness to pay for treatment. J Ment Health Policy Econ 2007;10: 73-85.

52. McIntosh E. Using discrete choice experiments within a cost-benefit analysis framework: some considerations. Pharmacoeconomics 2006;24:855-868.

53. Bryan S, Buxton M, Sheldon R, Grant A. Magnetic resonance imaging for the investigation of knee injuries: an investigation of preferences. Health Econ 1998;7:595-603.

54. Sassi F, McDaid D, Ricciardi W. Conjoint analysis of preferences for cardiac risk assessment in primary care. Int J Technol Assess Health Care 2005;21:211-218.

55. Giacomini M, Miller F, O'Brien BJ. Economic considerations for health insurance coverage of emerging genetic tests. Community Genet 2003;6:61-73.

56. Carlson JJ, Henrikson NB, Veenstra DL, Ramsey SD. Economic analyses of human genetics services: a systematic review. Genet Med 2005;7:519-523.

57. Rogowski W. Genetic screening by DNA technology: a systematic review of health economic evidence. Int J Technol Assess Health Care 2006;22:327-337.

58. Nielsen R, Gyrd-Hansen D. Prenatal screening for cystic fibrosis: an economic analysis. Health Econ 2002;11:285-299.

59. Doyle NM, Gardner MO. Prenatal cystic fibrosis screening in Mexican Americans: an economic analysis. Am J Obstet Gynecol 2003;189:769-774.

60. Tengs TO, Berry DA. The cost effectiveness of testing for the BRCA1 and BRCA2 breast-ovarian cancer susceptibility gene. Dis Manage Clin Outcomes 2000;2:15-24. 


\section{Grosse et al.}

61. Grann VR, Jacobson JS, Thomason D, Hershman D, Heitjan DF, Neugut AI. Effect of prevention strategies on survival and quality-adjusted survival of women with BRCA1/2 mutations: an updated decision analysis. J Clin Oncol 2002;20:2520-2529.

62. Asch DA, Patton JP, Hershey JC. Knowing for the sake of knowing: the value of prognostic information. Med Decis Making 1990;10:47-57.

63. Mushlin AI, Mooney C, Grow V, Phelps CE. The value of diagnostic information to patients with suspected multiple sclerosis. Rochester-Toronto MRI Study Group. Arch Neurol 1994;51:67-72.

64. Grann VR, Jacobson JS, Sundararajan V, Albert SM, Troxel AB, Neugut AI. The quality of life associated with prophylactic treatments for women with BRCA1/2 mutations. Cancer J Sci Am 1999;5:283-292.

65. Cappelli M, Surh L, Humphreys L, et al. Measuring women's preferences for breast cancer treatments and BRCA1/BRCA2 testing. Qual Life Res 2001;10:595-607.

66. Nelson HD, Huffman LH, Fu R, Harris EL; U.S. Preventive Services Task Force. Genetic risk assessment and BRCA mutation testing for breast and ovarian cancer susceptibility: systematic evidence review for the U.S. Preventive Services Task Force. Ann Intern Med 2005;143:362-379.

67. Almqvist EW, Brinkman RR, Wiggins S, Hayden MR; Canadian Collaborative Study of Predictive Testing. Psychological consequences and predictors of adverse events in the first 5 years after predictive testing for Huntington's disease. Clin Genet 2003; 64:300-309.

68. Holt K. What do we tell the children? Contrasting the disclosure choices of two HD families regarding risk status and predictive genetic testing. J Genet Couns 2006;15: 253-265.

69. Anderson K, Jacobson JS, Heitjan DF, et al. Cost-effectiveness of preventive strategies for women with a BRCA1 or a BRCA2 mutation. Ann Intern Med 2006;144:397-406.

70. Syngal S, Weeks JC, Schrag D, Garber JE, Kuntz KM. Benefits of colonoscopic surveillance and prophylactic colectomy in mutation carriers for hereditary nonpolyposis colorectal cancer. Ann Intern Med 1998;129:787-796.

71. Sommers BD, Beard CJ, D'Amico AV, et al. Decision analysis using individual patien preferences to determine optimal treatment for localized prostate cancer. Cancer 2007; 110:2210-2217.
72. Lee JT, Bridges JF, Shockney L. Can pharmacoeconomics and outcomes research contribute to the empowerment of women affected by breast cancer? Exp Rev Pharmacoeconomics Outcomes Res 2008;8:73-79.

73. Mushlin AI, Mooney C, Holloway RG, Detsky AS, Mattson DH, Phelps CE. The cost-effectiveness of magnetic resonance imaging for patients with equivocal neurological symptoms. Int J Technol Assess Health Care 1997;13:21-34.

74. Van Bebber SL, Liang SY, Phillips KA, Marshall D, Walsh J, Kulin N. Valuing personalized medicine: willingness to pay for genetic testing for colorectal cancer risk. Personal Med 2007;4:341-350

75. Cairns J, Shackley P, Hundley V. Decision making with respect to diagnostic testing: a method of valuing the benefits of antenatal screening. Med Decis Making 1996;16: 161-168.

76. Donaldson C, Shackley P, Abdalla M. Using willingness to pay to value close substitutes: carrier screening for cystic fibrosis revisited. Health Econ 1997;6: 145-159.

77. Donaldson C, Shackley P, Abdalla M, Miedzybrodzka Z. Willingness to pay for antenatal carrier screening for cystic fibrosis. Health Econ 1995;4:439-452.

78. Shackley P. Economic evaluation of prenatal diagnosis: a methodological review. Prenat Diagn 1996;16:389-395.

79. Bishop AJ, Marteau TM, Armstrong D, et al. Women and health care professionals preferences for Down's Syndrome screening tests: a conjoint analysis study. BJOG 2004;111:775-779.

80. Wilson B, Ryan M, Haites N. Assessing user preferences for, and costs of, genetic counselling for familial cancer risk in Scotland: a cost-utility analysis using conjoint analysis. London: Chief Scientist Office, 1999.

81. Hall J, Fiebig DG, King MT, Hossain I, Louviere JJ. What influences participation in genetic carrier testing? Results from a discrete choice experiment. J Health Econ 2006;25:520-537.

82. Marshall DA, Johnson FR, Phillips KA, Marshall JK, Thabane L, Kulin NA. Measuring patient preferences for colorectal cancer screening using a choice-format survey. Value Health 2007;10:415-430. 